\title{
Does Symphysis Pubis Width Change During Labor?
}

\author{
Buğra Çoşkun ${ }^{1}$, Demet Kokanalı², Burcu Timur ${ }^{3}$, Bora Çoşkun ${ }^{1}$, Ramazan Erda Pay ${ }^{4}$, Ulaş Fidan ${ }^{4}$, Kazım $^{2}$ \\ Emre Karaşahin ${ }^{4}$ \\ ${ }^{1}$ Obstetrics and Gynecology Department, Yüksek İhstisas Universty, Ankara, Turkey \\ ${ }^{2}$ Obstetrics and Gynecology Department, Health Science University Bilkent City Hospital, Ankara, Turkey \\ ${ }^{3}$ Obstetrics and Gynecology Department, Ordu University Research and Education Hospital, Ordu, Turkey \\ ${ }^{4}$ Obstetrics and Gynecology Department, Gülhane Education and Research Hospital, Ankara, Turkey
}

Received: 30 September 2019, Accepted: 20 November 2019, Published online: 31 December 2019

(C) Ordu University Institute of Health Sciences, Turkey, 2019

\begin{abstract}
Objectives: The anatomical and dynamic structure of the pelvis is an important element in delivery. During pregnancy, high progestorene and relaxin levels produce physiological ligament relaxation on the pelvis. The suitability of pelvic diameters is important in performing vaginal delivery. In this study we aimed to determine whether there is any change in symphysis pubis width during delivery.

Methods: This prospective observational study included 108 pregnant women who were at 37-42 weeks of gestation with cephalic presentation of a singleton pregnancy and were hospitalized for delivery. Symphysis pubis width was measured by transabdominal ultrasonography in each woman during the latent phase while cervical dilatation was $2 \mathrm{~cm}$, the active phase while cervical dilatation was $6 \mathrm{~cm}$ and the second stage within one hour of complete cervical dilatation.

Results: Symphysis pubis width value significantly increased as the labor progressed. Namely, the mean symphysis pubis width value measured in the latent phase was the smallest while the mean value in the second stage of the labor was the highest. In addition, birth weight of newborn was positively correlated each of symphysis pubis width values measured at all three different times.

Conclusion: Symphysis pubis width increases as labor progresses. And this increase advances as the weight of newborn increases.
\end{abstract}

Key words: Symphysis pubis; ultrasonography; labor

Suggested Citation: Coskun B, Demet Kokanali D, Timur B, Coskun B, Erdal Pay R, Fidan U, Karasahin KE. Does symphysis pubis width change during labor? Middle Black Sea Journal of Health Science, 2019; 5(3):221-225.

\section{Address for correspondence/reprints:}

Buğra Coskun

Telephone number: +90 (532) 3501404

E-mail: drbugracoskun@gmail.com

DOI: $10.19127 / \mathrm{mbsjohs} .627302$

\section{Introduction}

The anatomical and dynamic structure of the pelvis is an important element in delivery. Therefore, in obstetric practice, during vaginal delivery, it is always evaluated in optimal conditions for a safe and successful delivery (Gruss and Schmitt, 2015)

The joints between the bones that make up the pelvis are hard and strong joints and have minimal mobility. This allows the pelvis to form a solid structure and fully support the human skeleton (Lewis et al., 2017). Furthermore, the suitability of pelvic diameters is important in performing vaginal delivery (Maharaj, 2010). Namely, a small 
expansion of pelvic diameters is needed for the safe progression of vaginal delivery. Therefore, there may be some changes in the structure of pelvis during pregnancy. Changes in pregnancy and especially with hormonal effects, softening and loosening of the symphysis pubis (SP) and sacroiliac joints positively contribute to the progression of labor. This adaptation is characteristic for the harmony between fetus and pelvic diameters. The mechanism by which pelvic change occurs at birth is still not fully understood (Pavličev et al., 2019). And even the existence of such a change is debatable (Garagiola et al., 1989; Björklund et al., 1997).

In our study, we aimed to determine whether there is any change in SP width (SPW) during delivery and the factors associated with this condition if there is a change, as well.

\section{Methods}

This prospective observational study was performed at Gulhane Research and Education Hospital, Ankara, Turkey, between January 16, 2018, and June 25, 2018. The study protocol was approved by the Local Ethics Committee (Decision numbered 18/176). Written informed consent was obtained from all participants by the investigator (BC).

The women who had a singleton pregnancy at 37-42 weeks of gestation with cephalic presentation and were hospitalized for birth were included in the study. All women were Caucasian. Women with a prior cesarean delivery or any uterine operation, any SP separation history, or current symphysis joint pain were excluded.

Ultrasound examination was performed using a Voluson E10 ultrasound machine with a $3.5-\mathrm{MHz}$ trans-abdominal transducer (GE Healthcare, Chicago, USA). SPW was assessed transabdominally (Figure 1), as described by Rustamova et al. (Rustamova et al., 2009) in each woman between the uterine contractions during the latent phase while cervical dilatation was $2 \mathrm{~cm}$, the active phase while cervical dilatation was $6 \mathrm{~cm}$ and the second stage within one hour of complete cervical dilatation. The labor stages were determined according to Friedman's criteria. For the assessment of SPW, transabdominal transducer was vertically placed to the symphysial joint's upper border and then angled toward the lower abdomen, while the women were in supine position and her hips were flexed about $15^{\circ}$. So, a clear view of the upper part of the symphyseal joint was obtained in all women. The narrowest distance between the pubic bones where the medial pubic surfaces become parallel were measured as SPW. All the measurements were performed by senior author (KEK). To assess the reliability of the results, an experienced sonographer, who was blinded to study protocol and senior author's (KEK) results, repeated the measurements of the first 10 women. Test-retest series for the results in the first 10 women were done and showed good reliability.



Figure 1. Demonstration of symphysis pubis joint measurement

The demographic and obstetric data including woman's age, weight, length, gravida, parity, gestational age, ultrasonographic biparietal diameter (BPD) measurement of fetus in admission, birth weight of newborn, duration of active phase, the time between the full cervical dilatation and SPW measurement during second phase were recorded and entered into a statistical data base (SPSS, Version 17.0; Chicago, IL, USA) for analysis Kolmogorov-Smirnov test was used to analyze the normality of distribution of variables. Continuous variables with normal distribution are presented as mean \pm standard deviation. Median (minimum-maximum) value is used where normal distribution is absent. Quantitative variables are given as number (percentage). One-way repeated measures analysis of variance (ANOVA)was used to analyze differences of the measurements obtained at the three intervals during labor. Tukey test was used to determine the differences between the individual groups in ANOVA. Pearson correlation coefficients were analyzed to evaluate the correlation between the measurements of SP and other continuous variables, including woman's age, weight, length, body mass index (BMI), gestational age, ultrasonographic BPD measurement in admission, birth weight of newborn, duration of active phase, the time between the full cervical 
dilatation and SPW measurement during second phase. $\mathrm{P}<0.05$ was accepted to be statistically significant.

\section{Results}

This study included 108 pregnant women who had been hospitalized for delivery. Table I shows the characteristics of them. The mean age and BMI were $29.71 \pm 5.45$ years and $24.81 \pm 3.48 \mathrm{~kg} / \mathrm{m} 2$, respectively. Sixty-six $(61.11 \%)$ women were at nulliparous, while $23(21.30 \%)$ were primiparous and $19(17.59 \%)$ were multiparous. The mean ultrasonographic measurement of fetal BPD in admission and birth weight of newborn were $93.66 \pm 8.72 \mathrm{~mm}$ and $3580.19 \pm 358.23 \mathrm{~g}$, respectively. The calculated length of active phase has a mean value of $524.07 \pm 254.99$ minutes, while the mean time between the full cervical dilatation and SPW measurement in the 2nd stage of labor was $25.59 \pm 12.69$ minutes.
In Table 2, the results of SPW measurement were shown. According to these, each value measured at 3 different times during delivery was statistically different from each other (All $p$ values were $<0.05$ ). The mean SPW value measured in the latent phase $(5.89 \pm 1.32 \mathrm{~mm})$ was the smallest while the mean value in the second stage of the labor $(7.59 \pm 1.55 \mathrm{~mm})$ was the highest. In other words, the SPW value increased as the labor progressed.

In Table 3, the correlations between SPW measurements and other clinical factors were evaluated. As a result, it was found that only the birth weight was positively correlated each of SPW values measured at all three different times (for latent phase $r=0.24, p=0.01$; for active phase $r=0.26$, $\mathrm{p}=0.01$; for second stage $\mathrm{r}=0.25, \mathrm{p}=0.01$; respectively). There was no correlation with other factors.

Table 1. Characteristics of the participants

\begin{tabular}{lc}
\hline Variables & $\mathbf{N = 1 0 8}$ \\
\hline Woman's age (years) & $29.71 \pm 5.45$ \\
\hline Woman's length $(\mathrm{cm})$ & $165.18 \pm 5.76$ \\
\hline Woman's weight $(\mathrm{kg})$ & $67.70 \pm 10.08$ \\
\hline BMI $\left(\mathrm{kg} / \mathrm{m}^{2}\right)$ & $24.81 \pm 3.48$ \\
\hline Gravida (number) & $1(1-5)$ \\
\hline Parity (number) & $0(0-4)$ \\
multiparous & $19(17.59)$ \\
primiparous & $23(21.30)$ \\
nulliparous & $66(61.11)$ \\
\hline Gestational age at delivery (days) & $281.06 \pm 7.89$ \\
\hline Biparietal diameter measurement (mm) & $93.66 \pm 8.72$ \\
\hline Birth weight of newborn (g) & $3580.19 \pm 358.23$ \\
\hline Duration of active phase (minutes) & $524.07 \pm 254.99$ \\
\hline SPW measurement time in the 2nd stage of labor (minutes) & $25.59 \pm 12.69$ \\
\hline Variables were given as mean \pm standard deviation, median (minimum-maximum) & \\
\hline BMI: Body mass index, SPW: Symphysis pubis width & \\
\hline
\end{tabular}

Table 2 Comparison of symphysis pubis width measurements

\begin{tabular}{|c|c|c|c|c|c|c|c|}
\hline & $\begin{array}{l}\text { Latent phase } \\
\text {-I- }\end{array}$ & $\begin{array}{c}\text { Active phase } \\
\text {-II- }\end{array}$ & $\begin{array}{l}\text { The second stage } \\
\text {-III- }\end{array}$ & $\mathrm{P}$ & $\begin{array}{c}\mathrm{P}_{1} \\
\text { (I-II) }\end{array}$ & $\begin{array}{c}\mathrm{P}_{2} \\
(\mathrm{I}-\mathrm{III})\end{array}$ & $\begin{array}{c}\mathrm{P}_{3} \\
\text { (II-IV) }\end{array}$ \\
\hline SPW (mm) & $5.89 \pm 1.32$ & $6.53 \pm 1.42$ & $7.59 \pm 1.55$ & $<0.001$ & $<0.001$ & $<0.001$ & $<0.001$ \\
\hline $\begin{array}{l}\text { Values were } \\
\text { SPW: Symp } \\
\mathrm{P}<0.05 \text { was }\end{array}$ & $\begin{array}{l}\text { given as mean } \\
\text { ysis pubis wid } \\
\text { tatistically sigı }\end{array}$ & $\begin{array}{l}\text { = standard dev } \\
\text { ificant }\end{array}$ & & & & & \\
\hline
\end{tabular}


Table 3. Correlations between clinical factors and SPW measurement

\begin{tabular}{|c|c|c|c|c|c|c|}
\hline & \multicolumn{2}{|c|}{ Latent phase SPW } & \multicolumn{2}{|c|}{$\begin{array}{l}\text { Active phase } \\
\text { SPW }\end{array}$} & \multicolumn{2}{|c|}{$\begin{array}{c}\text { The second stage } \\
\text { SPW }\end{array}$} \\
\hline & $\mathrm{r}$ & $\mathrm{p}$ & $\mathrm{r}$ & $\mathrm{p}$ & $\mathrm{r}$ & $\mathrm{p}$ \\
\hline Woman's age & 0.06 & 0.57 & 0.07 & 0.48 & 0.11 & 0.25 \\
\hline Woman's length & 0.01 & 0.92 & 0.02 & 0.82 & 0.04 & 0.68 \\
\hline Woman's weight & 0.12 & 0.21 & 0.09 & 0.34 & 0.08 & 0.40 \\
\hline BMI & 0.12 & 0.20 & 0.09 & 0.36 & 0.07 & 0.47 \\
\hline Gestational age at delivery & 0.17 & 0.08 & 0.19 & 0.06 & 0.17 & 0.07 \\
\hline BPD measurement & 0.05 & 0.61 & 0.02 & 0.81 & 0.01 & 0.91 \\
\hline Birth weight of newborn & 0.24 & $\mathbf{0 . 0 1}$ & 0.26 & 0.01 & 0.25 & 0.01 \\
\hline Duration of active phase & 0.03 & 0.75 & -0.11 & 0.25 & -0.30 & 0.11 \\
\hline Measurement time in the 2 nd stage & 0.10 & 0.30 & 0.12 & 0.22 & 0.13 & 0.19 \\
\hline $\begin{array}{l}\text { BMI: Body mass index, BPD: Bipa } \\
\text { r: Pearson's correlation coefficient } \\
\mathrm{P}<0.05 \text { was statistically significant }\end{array}$ & $\mathrm{W}: \mathrm{Sy}$ & ysis $p$ & width & & & \\
\hline
\end{tabular}

\section{Discussion}

SP is a non-synovial joint connecting both pubic bones at midline in the anterior part of the pelvis and forms the anterior arch of the skeletal pelvis. It is interposed with a cartilaginous disc and supported by superior and inferior pubic ligaments. Its movements are extremely limited. However, SP can be more flexible and wider during pregnancy and labor, and after giving birth it returns to its former form (Alicioglu et al., 2008). In our study, the width of SP increased as labor progressed. In fact, this width was the least in the latent phase, and the highest in the second stage of labor. Our results are consistent with those of other studies who suggested that the SP may widen during labor (Björklund et al., 1997; Björklund et al., 1999; Rustamova et al., 2009). This may be an indicative of a physiological process. Labor is a process that occurs as a result of the appropriateness of certain characteristics of the mother and fetus. The fetus should be properly located in the birth canal and the fetus should be advanced to the outlet with sufficient maternal force. In addition, the pelvic structure must be suitable for delivery in order to achieve successful labor (Reitter et al., 2014). Increased SPW in the pelvis may also be an adaptation that widens pelvis and facilitates delivery.

Radiographic examinations have been used in similar very old studies and controversial results have been reported regarding the change of SPW during labor. It has been reported that this distance may increase or not change or even decrease (Thorp and Fray, 1938; Young, 1940). However, in more recent studies, it has been stated that ultrasonographic measurements can be used in the evaluation of SPW and this evaluation can be as reliable as radiographic evaluation (Becker et al., 2014). As a result of the use of ultrasonographic measurements, the idea that the width of SP increased during labor was accepted (Rustamova et al., 2009; Pavličev et al., 2019). However, it has been reported that these increase amounts are not very high (Rustamova et al., 2009). The results of our study supported this view and the amount of enlargement in SPW with the progression of labor differed by millimeters. During radiographic examination, correcting for magnification in images is difficult, because it is influenced by the distance of the measured object from the film. Therefore, examinations cannot be performed with subjects in a standardized position. Different postures may alter object-film distances, and change SP measurements. On the other hand, ultrasonographic examination ensures that the subjects' positions are standardized, is free of any risk and easy to use. Considering the low number of studies with ultrasonography in this topic, we think that our study may be useful for the literature.

When the relationship between SPW and various factors of labor was examined, we found that there was a positive correlation between SPW value and birth weight of the newborn for each period of labor. To the best of our knowledge, this finding is a new information for the literature Although this may support the presence of physiological changes in SPW to facilitate delivery as the weight of the newborn increases, it should be kept in mind that this may also be the result of increased mechanical load in the pelvis. We believe that further studies are needed to clarify this issue.

Our study has some limitations. The population in our study was relatively small and consisted of similar ethnic groups. We also did not evaluate the subjects' pelvic types in our study. Therefore, we were unable to determine whether genetical and 
ethnical factors and pelvic structure may influence changes in the SPW during labor.

\section{Conclusion}

As a result, SPW increases as labor progresses. And this increase advances as the weight of newborn increases. These findings suggest that SP is a dynamic entity that facilitates labor. Nevertheless, further studies are needed to fully understand the effects of labor on SP and the effects of SP on labor.

\section{Ethics Committee Approval:}

Ethics committee approval was received for this study from Clinical Research Ethics Committee of Health Science University. (26/06/2018- Toplant1 No:2018/9, Karar No:18/176)

Peer-review: Externally peer-reviewed.

Author Contributions: Author Contributions: Concept -BÇ, UF; Design BÇ, BoÇ, UFN, ; Supervision- DK, UF; Materials - BÇ, BoÇ, REP; Data Collection and/or Processing - BÇ, BoÇ, REP; Analysis and/or Interpretation - DK, BT; Literature Review - BÇ, BoÇ, BT; Writing - BÇ, DK, Critical Review - K EK

Conflict of Interest: No conflict of interest was declared by the author.

Financial Disclosure: The authors declared that this study hasn't received no financial support.

\section{References}

Alicioglu B, Kartal O, Gurbuz H, Sut N. 2008. Symphysis pubis distance in adults: a retrospective computed tomography study. Surgical and radiologic anatomy 30:153-157.

Becker I, Stringer MD, Jeffery R, Woodley SJ. 2014. Sonographic anatomy of the pubic symphysis in healthy nulliparous women. Clinical Anatomy 27:1058-1067.

Björklund K, Lindgren P, Bergström S, Ulmsten U. 1997. Sonographic assessment of symphyseal joint distention intra partum. Acta obstetricia et gynecologica Scandinavica 76:227-232.

Björklund K, Nordström M-L, Bergström S. 1999. Sonographic assessment of symphyseal joint distention during pregnancy and postpartum with special reference to pelvic pain. Acta obstetricia et gynecologica Scandinavica 78:125-130.

Garagiola DM, Tarver RD, Gibson L, Rogers RE, Wass JL. 1989. Anatomic changes in the pelvis after uncomplicated vaginal delivery: a CT study on 14 women. American Journal of Roentgenology 153:1239-1241.
Gruss LT, Schmitt D. 2015. The evolution of the human pelvis: changing adaptations to bipedalism, obstetrics and thermoregulation. Philosophical Transactions of the Royal Society B: Biological Sciences 370:20140063.

Lewis CL, Laudicina NM, Khuu A, Loverro KL. 2017. The human pelvis: variation in structure and function during gait. The Anatomical Record 300:633-642.

Maharaj D. 2010. Assessing cephalopelvic disproportion: back to the basics. Obstetrical \& gynecological survey 65:387-395.

Pavličev M, Romero R, Mitteroecker P. 2019. Evolution of the human pelvis and obstructed labor: New explanations of an old obstetrical dilemma. American Journal of Obstetrics \& Gynecology.

Reitter A, Daviss B-A, Bisits A, Schollenberger A, Vogl T, Herrmann E, Louwen F, Zangos S. 2014. Does pregnancy and/or shifting positions create more room in a woman's pelvis? American journal of obstetrics and gynecology 211:662. e661-662. e669.

Rustamova S, Predanic M, Sumersille M, Cohen WR. 2009. Changes in symphysis pubis width during labor. Journal of perinatal medicine 37:370-373.

Thorp DJ, Fray WE. 1938. The pelvic joints during pregnancy and labor. Journal of the American Medical Association 111:1162-1166.

Young J. 1940. Relaxation of the pelvic joints in pregnancy: pelvic arthropathy of pregnancy. BJOG: An International Journal of Obstetrics \& Gynaecology 47:493-524. 